\title{
Chromatin poises miRNA- and protein-coding genes for expression
}

\author{
Artem Barski, ${ }^{1,2}$ Raja Jothi, ${ }^{1,2,3}$ Suresh Cuddapah, ${ }^{1,2}$ Kairong Cui, ${ }^{1}$ Tae-Young Roh, ${ }^{1,4}$ \\ Dustin E. Schones, ${ }^{1}$ and Keji Zhao ${ }^{1,5}$ \\ ${ }^{1}$ Laboratory of Molecular Immunology, National Heart, Lung, and Blood Institute, National Institutes of Health, Bethesda, \\ Maryland 20892, USA
}

\begin{abstract}
Chromatin modifications have been implicated in the regulation of gene expression. While association of certain modifications with expressed or silent genes has been established, it remains unclear how changes in chromatin environment relate to changes in gene expression. In this article, we used ChIP-seq (chromatin immunoprecipitation with massively parallel sequencing) to analyze the genome-wide changes in chromatin modifications during activation of total human $\mathrm{CD}^{+} \mathrm{T}$ cells by T-cell receptor (TCR) signaling. Surprisingly, we found that the chromatin modification patterns at many induced and silenced genes are relatively stable during the short-term activation of resting $T$ cells. Active chromatin modifications were already in place for a majority of inducible protein-coding genes, even while the genes were silent in resting cells. Similarly, genes that were silenced upon T-cell activation retained positive chromatin modifications even after being silenced. To investigate if these observations are also valid for miRNA-coding genes, we systematically identified promoters for known miRNA genes using epigenetic marks and profiled their expression patterns using deep sequencing. We found that chromatin modifications can poise miRNA-coding genes as well. Our data suggest that miRNA- and protein-coding genes share similar mechanisms of regulation by chromatin modifications, which poise inducible genes for activation in response to environmental stimuli.
\end{abstract}

[Supplemental material is available online at http://www.genome.org. The raw and processed data from this study have been submitted to NCBI Short Read Archive (http://ncbi.nlm.nih.gov/sites/sra) under accession nos. SRPO0O2OI and SRPO00200 for resting cells, and to NCBI Gene Expression Omnibus (http://ncbi/nlm.nih.gov/geo) under accession no. GSE16657 for activated cells.]

Activation of T cells by T-cell receptor (TCR) signaling is a wellstudied physiological process (Kane et al. 2000). In living organisms, T-cell activation is initiated by TCR encountering a specific antigen presented by major histocompatibility complex (MHC) on the surface of antigen presenting cells, which triggers a cascade of signaling events leading to cell proliferation and expression of certain cytokines ( Janeway 2001). The process is accompanied by global chromatin decondensation and transcriptional activation of numerous genes, which is mediated by TCR signaling-activated kinases and transcription factors (Crabtree 1989; Chandok and Farber 2004).

Initiation of transcription involves binding of transcription factors to cis elements, followed by recruitment of coactivator enzymes, chromatin remodeling factors, mediators, and RNA polymerase (Li et al. 2007). Histone modifications and substitution of core histones by certain histone variants are believed to play an important role in this process (Weisbrod 1982; Jenuwein and Allis 2001; Stallcup 2001; Vermaak et al. 2003; Roh et al. 2005; Bernstein et al. 2007; Kouzarides 2007). Indeed, recent large-scale analyses indicate that specific chromatin modifications are enriched at ac-

\footnotetext{
${ }^{2}$ These authors contributed equally to this work.

Present addresses: ${ }^{3}$ Biostatistics Branch, National Institute of Environmental Health Sciences, National Institutes of Health, Research Triangle Park, NC 27709, USA; ${ }^{4}$ Department of Life Science, Pohang University of Science and Technology (POSTECH), Pohang 790-784, Republic of Korea.

${ }^{5}$ Corresponding author.

E-mail zhaok@nhlbi.nih.gov; fax (301) 480-0961.

Article published online before print. Article and publication date are at http://www.genome.org/cgi/doi/10.1101/gr.090951.109.
}

tive or silent promoters (Kim et al. 2005; Roh et al. 2006; Barski et al. 2007; Guenther et al. 2007; Heintzman et al. 2007; Mikkelsen et al. 2007; for review, see Bernstein et al. 2005, 2007; Schones and Zhao 2008).

The patterns of histone modifications on protein-coding genes in the human genome have been extensively characterized (Bernstein et al. 2005; Kim et al. 2005; Roh et al. 2006; Barski et al. 2007; Guenther et al. 2007; Mikkelsen et al. 2007; Wang et al. 2008; Kolasinska-Zwierz et al. 2009). However, very little is known regarding the regulation of miRNA genes, even though they have been implicated in various important biological functions (Bartel and Chen 2004; Pedersen and David 2008). While some miRNAs were shown to be transcribed by RNA polymerase III (Pol III) (Borchert et al. 2006), it is believed that a majority of miRNA genes require Pol II for expression (Lee et al. 2004; Chuang and Jones 2007). Most of the transcription start sites (TSSs) of miRNA genes were not known, although attempts have been made to identify TSSs of some individual miRNAs (Woods et al. 2007). A recent study suggests that histone modification patterns may be used to predict TSSs of miRNA genes (Marson et al. 2008).

We recently mapped the genome-wide distribution of many histone methylation (Barski et al. 2007) and acetylation marks (Wang et al. 2008), as well as nucleosome positions (Schones et al. 2008) in resting human $\mathrm{CD} 4^{+} \mathrm{T}$ cells. Among the 38 modifications examined, five modifications (H3K9me2, H3K9me3, H3K27me2, $\mathrm{H} 3 \mathrm{~K} 27 \mathrm{me} 3$, and H4K20me3) were associated with gene repression, whereas the others were associated with gene activation, or had no clear association (Wang et al. 2008). Consequently, the former modifications are considered "repressive" and the latter are 
considered "active." The specific association of histone modifications with transcriptional activity appears to confirm the notion that chromatin modifications regulate gene expression. However, even though the active modifications generally correlate with gene transcription, some of them have also been found at silent genes (Raisner et al. 2005; Roh et al. 2006; Barski et al. 2007; Guenther et al. 2007; Mikkelsen et al. 2007). Studies in yeast suggest that some histone modifications result from active transcription (Li et al. 2002; $\mathrm{Ng}$ et al. 2003) and have been proposed to be a memory of past transcriptional events ( $\mathrm{Ng}$ et al. 2003). On the other hand, experiments in mice have shown that changes in chromatin modifications might precede changes in gene expression (Chambeyron and Bickmore 2004).

To test if similar mechanisms exist in the human genome, we decided to examine changes in histone modifications in the genes induced or repressed by TCR signaling during activation of human $\mathrm{CD} 4^{+} \mathrm{T}$ cells. Surprisingly, we found that the induced genes were associated with the active modifications in resting $\mathrm{T}$ cells even before TCR signaling and, little or no change in histone modifications was detected upon gene induction in the short-term activation of T cells. This suggests that these inducible genes are poised for activation by chromatin in the resting cells. Furthermore, we identified promoters of miRNA genes using chromatin signatures and found that some silent miRNA genes in resting cells are also poised for expression by chromatin.

\section{Results}

\section{Changes in chromatin modifications and gene expression}

To examine if dynamic changes in chromatin modifications are associated with gene activation or repression, we combined gene expression analysis with the mapping of several chromatin modifications in resting and activated human $\mathrm{CD} 4^{+} \mathrm{T}$ cells. Total resting $\mathrm{CD}^{+}{ }^{\mathrm{T}}$ cells were isolated from blood to $95 \%-98 \%$ purity, and activated with anti-CD3/28 beads for $18 \mathrm{~h}$ resulting in the activation of $85 \%-95 \%$ of the cells (Supplemental Fig. S1A,B). The genome-wide distribution of eight histone methylation marks (H3K4me1, H3K4me3, H3K9me1, H3K27me1, H3K27me3, H3K36 me3, H3K79me2, and H4K20me1), histone variant H2A.Z, and RNA polymerase II (Pol II) in activated cells were mapped using ChIP-seq, as described previously (Barski et al. 2007) and compared with the distribution patterns of 29 histone modifications in resting $\mathrm{T}$ cells (Barski et al. 2007; Wang et al. 2008; Supplemental Table S1). At the same time, we profiled the mRNA levels using microarray analysis and analyzed miRNA profiles using deep sequencing. We found that 3244 protein-coding genes were constitutively expressed (see Methods) in both resting and activated cells (henceforth, referred to as $\mathrm{E} \rightarrow \mathrm{E}$ ), 5717 genes were constitutively silent in both cell states $(S \rightarrow S), 167$ genes were silent in resting cells, but expressed in activated cells $(\mathrm{S} \rightarrow \mathrm{E})$, and 271 genes were expressed in resting cells, but silenced upon activation $(\mathrm{E} \rightarrow \mathrm{S}$ ). The $\mathrm{S} \rightarrow \mathrm{E}$ subset was significantly enriched in genes associated with immune response, response to stimuli, and cell proliferation (Supplemental Table S2). Among the 541 known miRNAs in the human genome, 194 miRs were detected ( $>5$ tags, $P<10^{-13}$ based on a Poisson background model) in resting and/or activated T cells by deep sequencing (Supplemental Table S3).

Similar to our findings in resting T cells (Roh et al. 2006; Barski et al. 2007; Wang et al. 2008), the promoters of expressed genes in activated $\mathrm{T}$ cells were associated with high levels of Pol II and "active" histone modifications such as H3K4me1/3, H3K9me1, H3K27me1, H4K20me1, and histone variant H2A.Z. Many silent genes were associated with the repressive H3K27me3 modification.

Examination of the induced genes $(\mathrm{S} \rightarrow \mathrm{E}$ set) revealed that many of them already had active chromatin modifications at their promoters, while silent in the resting state. For example, the E2F1 gene, whose expression was not detected in resting $\mathrm{T}$ cells, but was induced upon T-cell activation, had peaks of active marks H3K4me3, H2A.Z, and H3K9me1, but not repressive mark H3K27me3 (Fig. 1A). Similar patterns were observed for LIF and CISH genes, which were induced upon TCR signaling (Supplemental Fig. S2). Thus, although these genes were not expressed, they had a chromatin environment resembling that of expressed genes, suggesting that their genomic loci were prepared for expression. Such genes that are currently not expressed, but possess "active" chromatin modifications will henceforth be referred to as "poised" genes. We also observed silent, but poised genes in activated T cells, especially among genes that were silenced upon T-cell activation. Many of these genes retained active chromatin modifications, even after being silenced. For example, the NKTR gene retained H3K4me3, H3K27me1, and H4K20me1 modifications, and histone variant H2A.Z at the promoter, even though it was silenced (Fig. 1B), suggesting that poising could be a result of previous transcription.

To investigate the poising of genes on a global scale, we calculated the average tag density profiles (see Methods) of histone modifications at promoter regions and in the gene bodies. As expected, constitutively expressed $(\mathrm{E} \rightarrow \mathrm{E})$ genes were enriched in active modifications, including H3K4me1/3, H3K9me1, H3K27me1, H4K20me1, and H2A.Z at their promoters and/or in the gene bodies in both resting and activated T cells, and had low
A
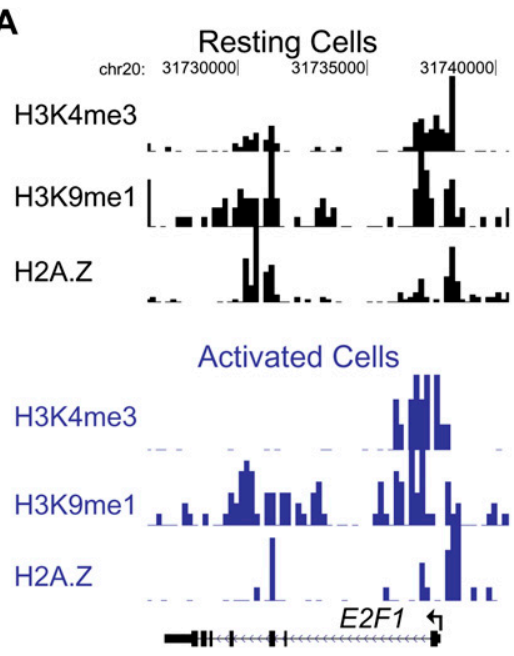

Figure 1. $E 2 F 1$ and NKTR genes do not change their chromatin status upon induction or silencing respectively. The chromatin modification patterns of the E2F1 gene $(A)$ and the NKTR gene $(B)$ are shown in resting (top panel) and activated (bottom panel) T cells.
B
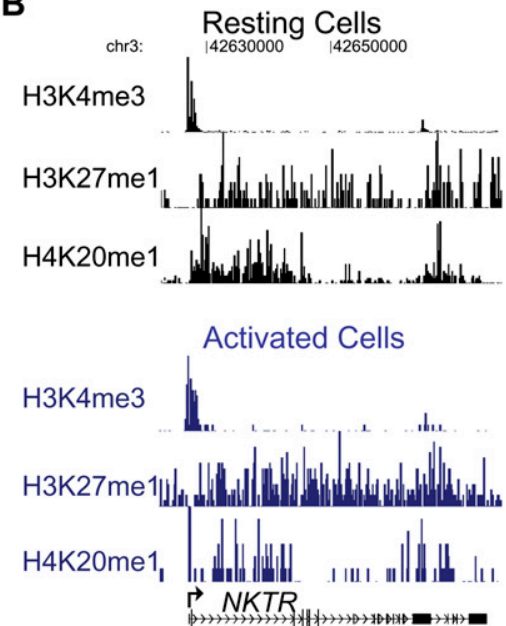
levels of the repressive modification H3K27me3 (Fig. 2; Supplemental Figs. S3, S4). In contrast, constitutively silent $(\mathrm{S} \rightarrow \mathrm{S})$ genes had lower levels of active modifications and higher levels of repressive H3K27me3. Interestingly, induced genes $(\mathrm{S} \rightarrow \mathrm{E})$ had high levels of active modifications not only in activated cells, where they were expressed, but also in resting cells, where they were silent. These genes had low levels of H3K27me3 in both cell states. Similarly, repressed genes $(\mathrm{E} \rightarrow \mathrm{S})$ possessed high levels of active modifications in both resting and activated T cells and low levels of H3K27me3. Pol II was present at a higher level in the bodies (Fig. 2C, arrow; Supplemental Fig. S5) of expressed genes compared to those of silent genes, thus confirming that the absence of mRNA product from silent genes was due to the absence of transcription. However, high levels of Pol II were present at
A

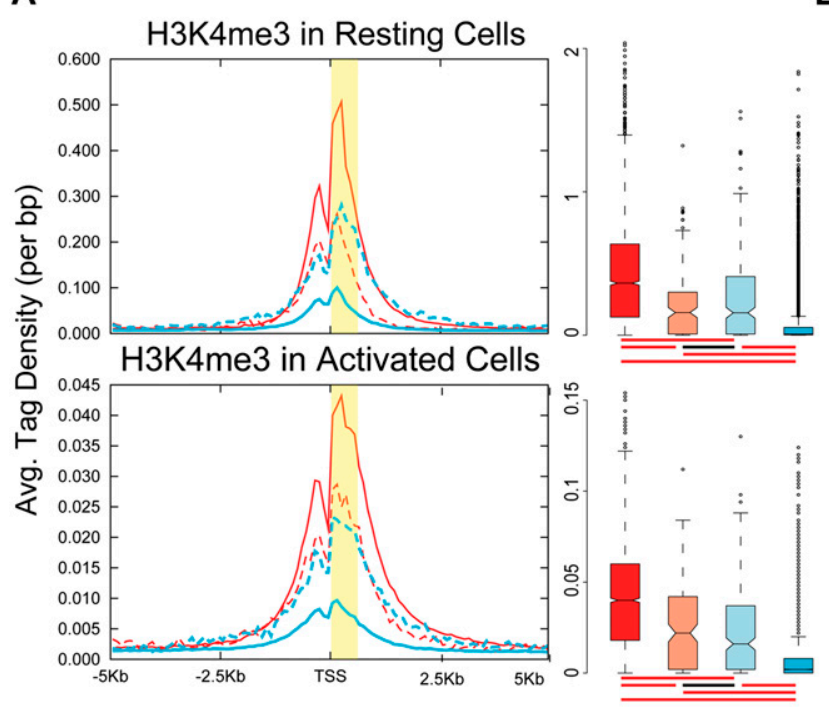

C

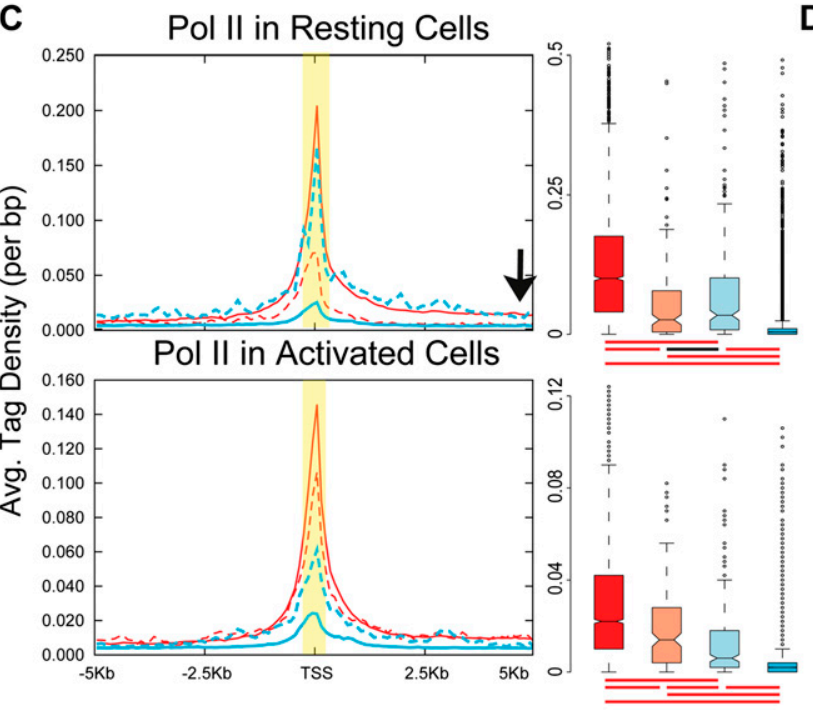

B

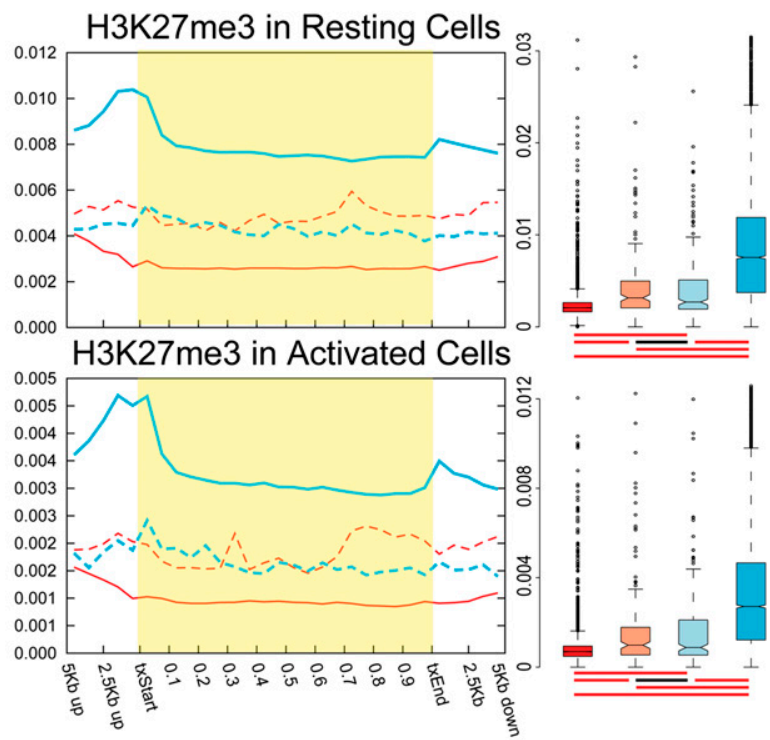

D

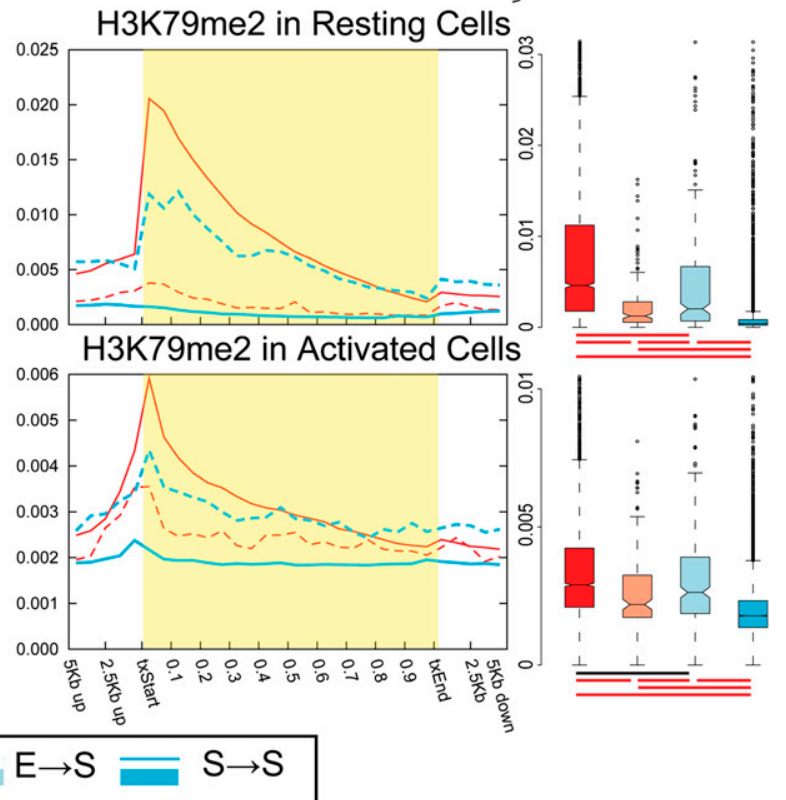

Figure 2. A majority of the genes do not change their chromatin status upon induction or silencing. Average ChIP-seq tag density for the four gene sets are shown in resting (top panel) and activated (bottom panel) $\mathrm{CD} 4^{+} \mathrm{T}$ cells. The four gene sets: $\mathrm{S} \rightarrow \mathrm{E}$ (silent in resting and expressed in activated T cells), red dashed line in density plot and light red in box plot; $\mathrm{E} \rightarrow \mathrm{S}$, blue dashed line and light blue; $\mathrm{E} \rightarrow \mathrm{E}$, red line and red; $\mathrm{S} \rightarrow \mathrm{S}$, blue line and blue. The average tag density values for resting and activated cells are not directly comparable as they have not been normalized across samples. Box plot summarizes the distribution of the number of tags in the region of interest (highlighted in yellow) for each gene set. Box plot captures the median, the middle $50 \%$ of the data points, and the outliers. The data points for each gene set are divided into quartiles, and the interquartile range (IQR) is calculated as the difference between the first and the third quartiles. The filled box denotes the middle $50 \%$ of the data points, with the horizontal line in-between and the notch representing the median and confidence intervals, respectively. Data points more than 1.5 times IQR lower or higher than first or third quartiles, respectively, represent outliers and are shown as dots. The horizontal line connected by vertical dashed lines above and below the filled box (whiskers) represents the largest and the smallest nonoutlier data points. The cluster of horizontal red and black lines below each box plot signifies whether or not the difference between the medians of two gene sets are statistically significant, respectively $(P<0.01$; two-tailed Wilcoxon rank-sum test). $(A) \mathrm{H} 3 \mathrm{~K} 4 \mathrm{me} 3,(B)$ H3K27me3, (C) Pol II, and (D) H3K79me2. Profiles for other modifications are presented in Supplemental Figures S3 and S4.

\section{Genome Research}

www.genome.org 
induced gene $(\mathrm{S} \rightarrow \mathrm{E})$ promoters in both resting and activated $\mathrm{T}$ cells. In addition, Pol II did not disappear from the repressed gene $(\mathrm{E} \rightarrow \mathrm{S})$ promoters after the genes were silenced in activated T cells.

The methylation of histone H3K36 and H3K79 residues in the gene body is considered to be associated with active transcription (Li et al. 2002, 2007; Krogan et al. 2003; Steger et al. 2008). However, we detected increased levels of H3K36me3 in induced genes $(\mathrm{S} \rightarrow \mathrm{E})$ even in resting cells (Supplemental Fig. S3E), suggesting that this modification might have remained from previous rounds of transcription or be generated in a transcription-independent manner. We found that only H3K79me2 was somewhat correlated with the gene expression status in resting cells. Upon T-cell activation, H3K79me2 levels increased in induced genes, but repressed genes $(\mathrm{E} \rightarrow \mathrm{S}$ ) did not lose the methylation (Fig. 2D), suggesting that longer times may be required to erase this modification. Our observations remained the same even when we used alternative gene sets defined using more stringent criteria (see Methods for details).

To ensure that the observed global trends are not due to a few genes with extremely high levels of histone modifications, we examined promoter modifications on a gene-by-gene basis. For each gene set, we calculated the percentage of genes possessing statistically significant $\left(P\right.$-value $\left.<10^{-3}\right)$ levels of H3K4me3, H2A.Z, and Pol II tags at gene promoters (Methods). We found H3K4me3 at $83 \%$ and $81 \%$ of constitutively expressed gene $(\mathrm{E} \rightarrow \mathrm{E})$ promoters in resting and activated T cells, respectively (Fig. 3A). Interestingly, $68 \%$ and $64 \%$ of induced genes $(\mathrm{S} \rightarrow \mathrm{E})$ had $\mathrm{H} 3 \mathrm{~K} 4 \mathrm{me} 3$ in resting and activated $\mathrm{T}$ cells, respectively. In fact, only three out of the 167 induced genes $(\mathrm{S} \rightarrow \mathrm{E})$ actually became H3K4me3-positive upon T-cell activation. Similarly, a majority of repressed genes $(\mathrm{E} \rightarrow \mathrm{S})$ had H3K4me3 in both resting (65\%) and activated (58\%) cells. Only 18 of the 271 repressed $(\mathrm{E} \rightarrow \mathrm{S})$ genes lost $\mathrm{H} 3 \mathrm{~K} 4 \mathrm{me} 3$ upon T-cell activation. Two-thirds of the constitutively silent genes $(\mathrm{S} \rightarrow \mathrm{S}$ ) lacked H3K4me3. We observed a largely similar pattern for H2A.Z and Pol II (Fig. 3B,C). While we found no changes in regard to the presence or absence of chromatin modifications and Pol II at promoters, their levels at some individual genes could have changed.

\section{Nature of poised genes}

Our data indicate that H3K4me3, as well as histone variant H2A.Z and Pol II, occupied promoters of $15 \%-30 \%$ of all silent genes. This is consistent with recent observations that a large fraction of silent genes had H3K4me3 at their promoters (Barski et al. 2007; Guenther et al. 2007; Mikkelsen et al. 2007). To better understand the nature of the poised genes, we performed Gene Ontology (GO) analysis of the silent genes having significant levels of Pol II at their promoters in resting cells. This analysis revealed that this gene set was enriched with functions related to metabolic processes and cell cycle (Supplemental Table S5). This was not surpris- ing given that the genes involved in basic cellular processes would have to be prepared for expression in case certain contingencies arise. When T cells receive a signal (TCR signaling being just one example), some of these genes begin to be expressed. One would expect the cell-type specific genes that are not needed in a given cell type not to be poised. Indeed, H3K4me3 and Pol II tag density profiles of silent genes involved in muscle or organ development, which are not needed for $\mathrm{T}$ cells, showed no enrichment in H3K4me3 or Pol II. Conversely, profiles for cell cycle or metabolismrelated silent genes showed high enrichment at the promoter (Fig. 3D,E).

\section{Systematic identification of miRNA gene promoters using chromatin signatures}

Our data above indicate that the majority of inducible proteincoding genes are poised by chromatin for possible future expression. Next, we sought to find if the poising mechanism also applies to miRNA-coding genes. Since most miRNAs are believed to be transcribed by Pol II (Lee et al. 2004; Chuang and Jones 2007), we hypothesized that their promoter structure and regulation may be similar to that of protein-coding genes. Indeed, we found that transcription of pri-miRNAs might be initiated from promoters that bear chromatin modifications similar to that of protein-coding genes. For example, we detected peaks of chromatin modifications including H3K4me3, H3K9ac, and H2A.Z, as well as Pol II upstream of a cluster of three highly expressed miRNA genes MIRLET7A1, MIRLET7F2, and MIRLET7D located in an intergenic region on chromosome 9 (Fig. 4A; Supplemental Table S3). High

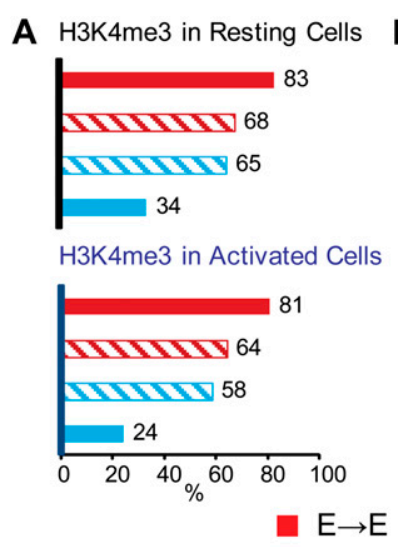

D

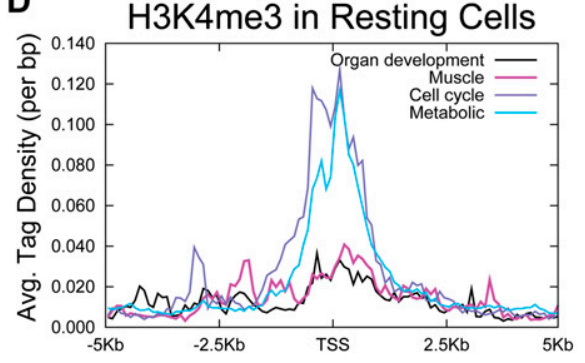

B H2A.Z in Resting Cells

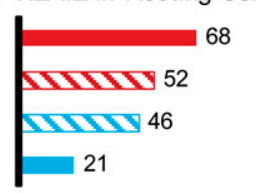

H2A.Z in Activated Cells

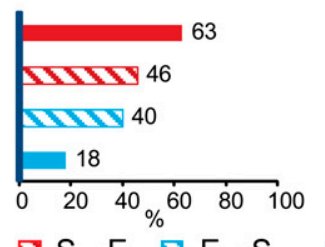

$\Delta \mathrm{S} \rightarrow \mathrm{E} \triangle \mathrm{E} \rightarrow \mathrm{S}$

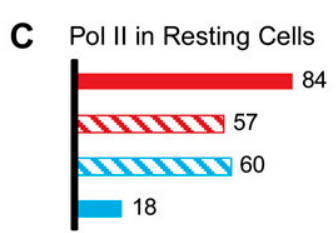

Pol II in Activated Cells

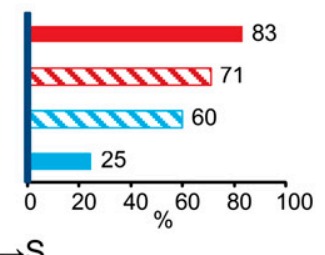

$\mathrm{S} \rightarrow \mathrm{S}$

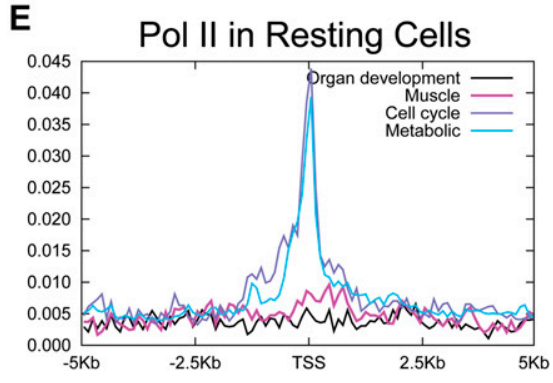

Figure 3. Inducible genes are poised for expression. $(A-C)$ A majority of inducible genes have H3K4me3 $(A)$, H2A.Z (B), and Pol II (C) at their promoters. The percentage of genes that have significant $\left(P\right.$-value $\left.<10^{-3}\right)$ number of tags at the promoter for each set of genes is shown in resting (top panel) and activated (bottom panel) T cells. $(D, E)$ Silent genes associated with cell cycle and metabolism, but not muscle or organ function and development, are poised in T cells. H3K4me3 (D) and Pol II (E) tag density profiles for silent genes taking part in specific biological processes. 
A

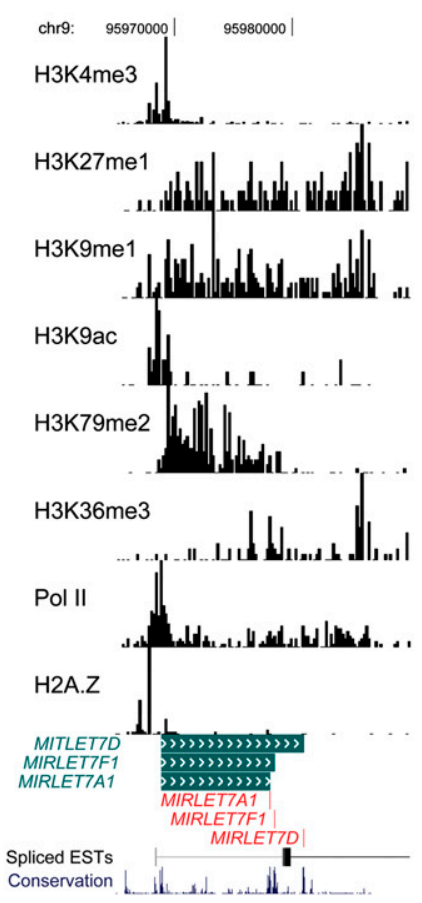

B

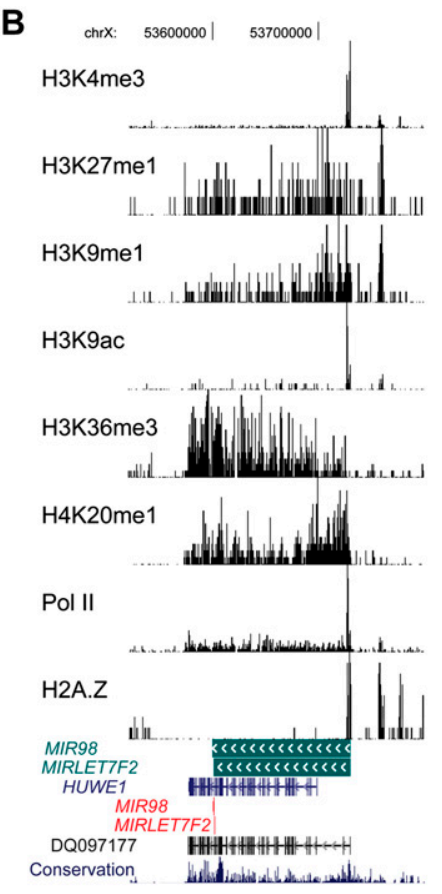

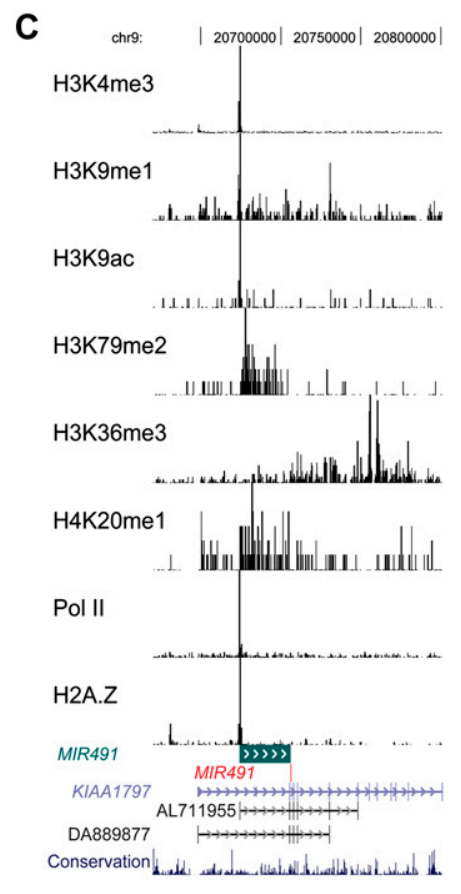

Figure 4. miRNA promoters have chromatin modification patterns similar to that of protein-coding genes. Chromatin modification patterns in the region surrounding the intergenic MIRLET7 miRNA cluster (A), and intragenic MIR98 and MIRLET7F2 (B), and MIR491 (C) are shown. Putative promoters are marked by H3K4me3, H2A.Z, and Pol II peaks. The green bars extend from the predicted transcription start site to the $3^{\prime}$ end of the pre-miRNA. H3K27me1, H3K9me1, H3K79me2, and H3K36me3 modifications, typically found within gene bodies of protein-coding genes, are seen within the putative miRNA coding transcript.

levels of H3K27me1, H3K9me1, H3K79me2, and H3K36me3, which are typically associated with actively transcribed regions of protein-coding genes, were found immediately downstream from these peaks. Presence of expressed sequence tags (ESTs) starting near the peaks and extending past the miRNAs provides further evidence of transcriptional initiation from the predicted TSS regions for the miRNA genes.

Based on these observations, we designed an algorithm that uses a kernel density estimation function (see Methods) to identify putative promoters for all known miRNAs using H3K4me3, H2A.Z, and Pol II data in resting cells. The algorithm starts from the $5^{\prime}$ end of the pre-miRNA, and traverses upstream until it finds statistically significant peaks for each of the three modifications. A promoter for a miRNA-coding gene is predicted if the peaks of at least two out of the three colocalize, a pattern typically observed at the promoters of protein-coding genes (see Methods for details). When this algorithm was tested to predict the promoters of 1000 highly expressed genes, $\sim 90 \%$ of the predicted promoters were within a few hundred base pairs of the annotated TSSs (Supplemental Fig. S6). Of the 541 human miRNAs in miRBase (Griffiths-Jones et al. 2006), we identified promoters for 234 miRNAs, 129 of which are located within a gene, and 105 of which are intergenic (Supplemental Table S3). About $67 \%$ either shared the promoter with a protein-coding gene that hosts the miRNA or had an EST initiating at the predicted promoter and extending past the miRNA, suggesting that the transcription of pri-miRNAs is indeed initiated from the promoters. Another 15\% had ESTs starting at the promoter, extending toward the miRNA, but ending short of it. Of the 85 intergenic miRNAs that did not share a promoter with a proteincoding gene, 36 had an EST starting at the predicted promoter and extending past the miRNA, and 19 had an EST starting at the promoter, but ending short of the miRNA (Fig. 4; Supplemental Table S3).

To ensure that the predicted miRNA promoters are accurate, we picked a few predicted promoters for which no EST support was available and performed validation experiments. First, we performed 5' RACE experiments to detect pri-miRNA transcripts downstream from predicted TSSs of seven clusters of miRNAs (16 miRNAs total) that were expressed in T cells (Supplemental Fig. S7B; see Methods). We were able to detect the pri-miRNA transcripts and their TSSs were within $\sim 200$ base pair (bp) of their predicted promoters (Supplemental Fig. S7). This resolution is similar to that achieved for a set of protein-coding genes (Supplemental Fig. S6), and is reasonable given that the resolution of the chromatin modification data used to predict the promoters was $\sim 150$ bp. Second, we used a promoter reporter assay to test the promoter activity at the predicted miRNA promoters. We cloned $\sim 650$ bp surrounding putative promoters of six miRNA genes that were not expressed in T cells into a pGL3 enhancer luciferase reporter vector containing an $S V 40$ enhancer, but no promoter. Two sequences that did not display Pol II binding and H3K4me3 enrichment in ChIP-seq were used as controls. When transfected into Jurkat cells, all six miRNA promoter constructs had significantly higher activity than controls $(P<0.005)$, thus confirming that predicted promoters indeed possess promoter activity (Fig. 5; see Methods). A chromatin-based approach to promoter prediction has also been used and validated by others (Marson et al. 2008; Guttman et al. 2009). A comparison of the miRNA promoters predicted by our approach with that predicted by Marson et al. (2008), which used H3K4me3 mark and DNA sequence features, revealed that a majority of our predictions are the same as that reported in this other study.

\section{Genome Research} www.genome.org 


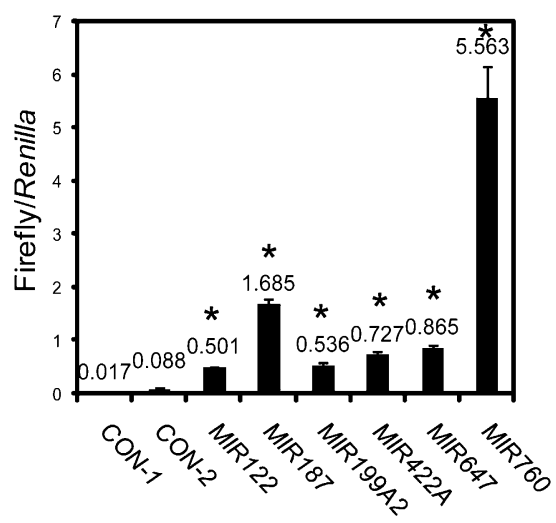

Figure 5. Promoter-reporter assay. Predicted miRNA promoters or control sequences were cloned into $\mathrm{pGL} 3$ enhancer vector containing SV40 enhancer, but no promoter. Plasmids were transfected into Jurkat cells together with Renilla luciferase control. Luciferase activity was measured after two days. Ratio of firefly and Renilla luciferase signal + SD $(n=3)$ is shown. ${ }^{*} P$-value $<0.005$ in Student $t$-tests vs. both controls 1 and 2 .

We found examples of intragenic miRNAs having independent promoters or sharing promoters with the protein-coding genes. For example, MIR98 and MIRLET7F2 located within the HUWE1 gene on chromosome X, had peak signals of Pol II, H3K4me3, H2A.Z, and other modifications $\sim 25 \mathrm{~kb}$ upstream of the annotated HUWE1 promoter with H3K36me3 extending from this putative promoter toward the miRNAs, suggesting that the miRNA transcription actually starts upstream of the host gene. ESTs were also found to start in this region (Fig. 4B). Another example of an intragenic miRNA starting downstream from the host gene's TSS is shown in Figure 4C. However, most of the predicted promoters for intragenic miRNAs were the same as those of the host genes (Supplemental Fig. S8).

To investigate the chromatin environment at miRNA promoters, we examined the average tag density profiles of chromatin modifications at these loci (Supplemental Fig. S9). While H3K4 me3, H2A.Z, and Pol II were used for promoter prediction and thus, one can expect to see their peaks at the promoters, the rest of the modifications were enriched at promoters independent of the prediction algorithm. In particular, H3K9me1, H4K20me1, and H3K36me3 were elevated downstream from the TSSs (Supplemental Fig. S9G,J,K). These results show that miRNA genes have a chromatin environment similar to that of protein-coding genes.

\section{Poising of miRNA genes by chromatin}

Since peaks of Pol II, H3K4me3, and H2A.Z in resting cells were used to predict miRNA promoters, the miRNAs (i.e., the pri-miRNAs coding for these miRNAs) whose transcription starts at these predicted promoters must be either expressed or poised in resting cells. To avoid a circular argument, instead of asking whether induced miRNA genes were poised in resting cells, we asked how many of the 234 predicted miRNA promoters (which, by definition, are associated with "active" modifications) were silent in resting cells. To account for the fact that two or more miRNAs often share the same promoter, we considered 175 promoters driving the expression of 234 miRNAs (Supplemental Table S3). Our deep sequencing of miRNAs indicated that $42 \%$ of these promoters had zero sequence reads mapped to their product miRNA(s), suggesting that the miRNAs coded by these transcripts are silent in resting cells. These silent miRNAs already contain active chromatin modifications at their promoters suggesting that they might be poised for future expression. Indeed, we found that several of these miRNA genes were induced by TCR signaling. For example, there were zero tags for intragenic MIR877 in resting T cells and 80 tags in activated T cells; for intergenic MIR301B, the expression increased from one to 11 tags. (Fig. 6; for more examples, see Supplemental Fig. S10). This suggested that chromatin modifications poise some miRNA genes for expression.

\section{Discussion}

We have provided evidence that the chromatin modification patterns at a majority of induced and repressed genes are stable during short-term activation of resting human $\mathrm{CD} 4^{+} \mathrm{T}$ cells. This suggests that changes in the chromatin environment of induced or repressed genes take a relatively long time. Given that, according to FRAP studies (Hager et al. 2006), chromatin states can be changed relatively fast, the stability of chromatin modification patterns we observe has to be purposefully maintained.

In general, as shown in this and other studies, active chromatin modifications were found to be associated with expressed genes. As suggested by Ruthenburg et al. (2007), the association between expressed genes and active modifications can be explained in four ways: histone modifying enzymes (e.g., histone methyl transferases [HMTs]) are recruited (1) by TFs before transcription initiation, which helps to recruit Pol II machinery; (2) by the polymerase machinery itself concurrently with or after initiation; (3) by other histone modifications via effector proteins; or (4) by RNA. However, we and others have found that some silent genes are also associated with active modifications including H3K4me3 (yeast [Ng et al. 2003], mouse [Mikkelsen et al. 2007], and human [Barski et al. 2007; Guenther et al. 2007]), H2A.Z (yeast [Raisner et al. 2005]) and human (this study), and other modifications (human [this study]). In this context, gene poising or presence of active chromatin marks at silent genes can be

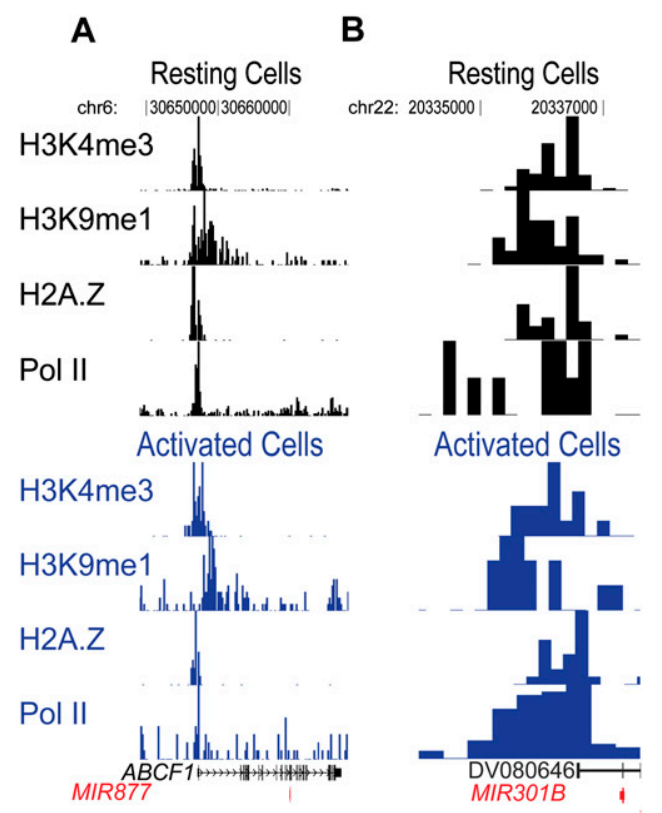

Figure 6. Inducible miRNA genes are poised for expression. The chromatin modification patterns at the intragenic MIR877 $(A)$ and intergenic $M I R 301 B(B)$ genes that are induced upon T-cell activation. 
explained by either de novo poising of silent genes for future expression (assuming recruitment mechanism 1 or 3 ), or as a memory of past transcription (mechanisms 2-4).

In yeast, the only known H3K4 HMT, Set1, is recruited to active genes via Ser5 phosphorylated Pol II, suggesting that the H3K4me3 mark remains at gene promoters mostly as a memory of past transcription (Ng et al. 2003). However, more evolutionarily advanced organisms, such as flies and mammals have several H3K4 HMTs. These are recruited not only by transcribing Pol II, but also by sequence-specific transcription factors, via interaction with other histone modifications, or even RNA (for review, see Ruthenburg et al. 2007). We found evidence suggesting both de novo poising and memory of past transcription in our experiments with human cells. While only $15 \%-20 \%$ of all silent genes have active modifications, as many as $60 \%-70 \%$ of inducible silent genes are associated with active modifications and Pol II. This suggests that active modifications are deposited to poise inducible genes for future expression. At the same time, a majority of recently silenced genes retained the active modifications, suggesting the "memory of past transcription" principle. In this study, the only modification that can be clearly explained in only one way seems to be H3K79me2. We detected little enrichment of this modification at inducible genes before gene induction, but significant levels at the genes that were recently silenced, suggesting that this modification is deposited only during active transcription and its presence at silent genes is due to short-term "transcriptional memory."

It is important to note that our experiments have been performed using total $\mathrm{CD} 4^{+} \mathrm{T}$ cells, including both naive and memory $\mathrm{T}$ cells. Unlike naïve $\mathrm{T}$ cells that have never met an antigen, memory $\mathrm{T}$ cells have been activated by interaction with antigen, but returned to a quiescent, resting state. Therefore, the chromatin modifications at the inducible genes could reflect the TCR signaling history of memory T cells. Indeed, IL2, IFNG, and IL4 promoters are marked with $\mathrm{H} 3 \mathrm{~K} 4 \mathrm{me} 3$ in our cells. Our analysis of chromatin modifications in naïve $\mathrm{CD} 4^{+} \mathrm{T}$ cells in mouse (Wei et al. 2009) showed that several cytokine gene promoters, including Il2, Il4, and Ifng, were not marked by active modifications in naïve cells. This observation suggests that the poising we observed in total human $\mathrm{CD} 4^{+} \mathrm{T}$ cells could have come from memory cells, which have experienced transcription of certain cytokine genes due to TCR signaling in the past. On the other hand, poised genes related to general cellular machinery and cell cycle (e.g., E2F1, SRF) are poised in mouse even in naïve T cells. These genes have obviously been expressed in the past during T-cell development. This suggests that some genes expressed in the past can stay poised, possibly, to facilitate transcription in the future. When naïve $\mathrm{CD} 4^{+}$ $\mathrm{T}$ cells were differentiated to various T-helper cell lineages, significant changes of histone modification at critical cytokine and transcription factor genes were detected (Wei et al. 2009). Similarly, dramatic chromatin changes were also detected when hematopoietic stem cells were differentiated to erythrocyte precursor cells (Cui et al. 2009) and in other studies of cell differentiation (Mikkelsen et al. 2007; Mohn et al. 2008). In contrast, even though many genes were induced, the short-term TCR signaling of $\mathrm{CD} 4^{+} \mathrm{T}$ cells in this study did not cause dramatic changes in chromatin modifications. Altogether this leads us to hypothesize that execution of cell function, such as quick production of cytokines upon activation, is facilitated by preexisting chromatin modifications or, in other words, gene poising. At the same time longterm cell state changes, such as naïve to Th1/2 T-helper cell differentiation are accompanied by changes in chromatin modifications that serve as a robust epigenetic mechanism stabiliz- ing the changed cell states and supporting future execution of cell function. However, further experiments are required to validate this hypothesis.

In this work we analyzed only histone methylation and acetylation modifications. It is possible that other histone modifications, both known and undiscovered, can play a significant role in the regulation of gene expression. In addition to acetylations and methylations these might include phosphorylations, ubiquitinations, and other modifications. These remain interesting areas for future studies.

The poised genes are marked by active chromatin modifications and possess Pol II at their promoters, but are not transcribed, suggesting that recruitment of Pol II is not the final step in the regulation of transcription for a significant percentage of genes. The presence of paused Pol II at the promoters of silent genes has been observed in both the Drosophila and human (Barski et al. 2007; Guenther et al. 2007; Muse et al. 2007; Zeitlinger et al. 2007; Schones et al. 2008). While it is not clear what prevents Pol II from transcribing after its recruitment to promoter, transition from transcriptional initiation to elongation is known to be regulated by a P-TEFb complex (Core and Lis 2008; Steger et al. 2008). However, the manner of gene-specific recruitment of P-TEFb remains to be studied.

Our data suggest that chromatin modifications poise genes, which creates a chromatin environment that can accommodate transcriptional activation by specific transcription factors induced by TCR signaling or other environmental stimuli. We found that a poising mechanism is employed in the regulation of not only protein-coding, but also miRNA genes. This suggests that control of miRNA expression works similarly to that of protein-coding genes.

\section{Methods}

\section{Cells, antibodies, and ChIP-seq}

Human $\mathrm{CD}^{+} \mathrm{T}$ cells were prepared by negative selection as described previously (Barski et al. 2007). Activation was performed by addition of anti-CD3/anti-CD28 beads (Invitrogen) to cells for $18 \mathrm{~h}$ according to manufacturer's instructions. Chromatin was prepared by formaldehyde cross-linking and sonication for H3K79me2 and Pol II ChIPs and by micrococcal nuclease digestion for the rest of the ChIP experiments. ChIP-seq was performed as described previously (Barski et al. 2007; Wang et al. 2008; see also Barski and Zhao 2009; Cuddapah et al. 2009 for review and protocol, respectively). Antibodies used for ChIP are listed in Supplemental Table S1 and their specificity was assessed in a previous study (Wang et al. 2008).

\section{Illumina pipeline analysis}

Sequenced tags were mapped to the human genome (hg18) using the Illumina Analysis Pipeline. The mapped tags for each sample were converted to a browser extensible data (BED) file, detailing the genomic coordinate of each tag. Summary files, displaying the number of tags in 200-bp windows, in BED format were used for viewing in the UCSC Genome Browser, and to generate screenshots. The read numbers for each sample are listed in Supplemental Table S1.

\section{Gene Ontology analysis}

GO analysis was performed using DAVID functional annotation tool, using a $P$-value cutoff of 0.01 (http://david.abcc.ncifcrf.gov/) (Huang da et al. 2007). 


\section{Expression analysis and gene sets}

Total RNA was prepared using an RNeasy kit (Qiagen) and analyzed using Human Genome U133 Plus 2.0 array (Affymetrix). Data were previously deposited to GEO as GSE10437.The Affymetrix Microarray Suite 5 (MAS5.0) algorithm (Affymetrix 2002) was used on the expression data to make absent/present calls. Probes that were designated as present (or absent) across all replicates of a given sample were considered expressed (or silent, respectively) in that sample. Only those Affymetrix probe sets, which were unequivocally mapped to UCSC known genes (Hsu et al. 2006), were considered for further analysis, leaving us with 9399 genes for further analysis. Set $\mathrm{E} \rightarrow \mathrm{E}$ ( or $\mathrm{S} \rightarrow \mathrm{S}$ ) was defined as the set of genes expressed (or silent) in both resting and activated T cells. Genes that were silent in resting cells, but expressed in activated cells were defined as set $\mathrm{S} \rightarrow \mathrm{E}$, and those that were expressed in resting cells and silent in activated cells were defined as set $\mathrm{E} \rightarrow \mathrm{S}$.

To ensure that our observations do not hinge critically on the definition of the gene sets, and that they will hold even if one uses a more stringent definition for gene sets, we repeated the analyses using alternative gene sets defined based on (1) P/A calls, (2) the requirement that there is at least a twofold change in expression for genes switching states from $\mathrm{P}$ to $\mathrm{A}$ and $\mathrm{A}$ to $\mathrm{P}$ in all replicates, and (3) the requirement that the expression of genes in $S \rightarrow S$ are below the median expression levels in both cell states in all replicates, and that the expression of genes in $\mathrm{E} \rightarrow \mathrm{E}$ are above the median expression levels in both cell states. These restrictions reduced the number of genes in $\mathrm{E} \rightarrow \mathrm{E}, \mathrm{S} \rightarrow \mathrm{E}, \mathrm{E} \rightarrow \mathrm{S}$, and $\mathrm{S} \rightarrow \mathrm{S}$ sets from 3250, 168,271 , and 5729 to $2544,45,44$, and 4941 , respectively. The average tag density plots for these strictly defined gene sets revealed no changes in the observed trends (see Supplemental Fig. S11) compared to those plotted using gene sets defined using only A/P calls (Fig. 2; Supplemental Figs. S3, S4).

For miRNA analysis, RNA was prepared using miRNeasy kit (Qiagen). Cloning was performed by a protocol similar to that of Lau et al. (2001) followed by deep sequencing on Illumina genetic analyzer (A Barski, unpubl.). Read numbers corresponding to each human miRNA are shown in Supplemental Table S3.

\section{Chromatin modification profiles}

To examine modification patterns near the TSSs of each gene set, all genes in the set were aligned relative to their TSS coordinates. Tag density was calculated in 100-bp windows relative to the TSS, and the average tag density (used in the plots) denotes the average number of tags per base pair. Modification patterns in the gene bodies of each gene set were examined in a similar fashion, except that the genes were aligned relative to their TSS and transcription end site (TSS). The tag density within the gene body was calculated using windows, whose length is $5 \%$ of the gene length.

It should be noted that tag densities have not been normalized across cell states, and thus are not directly comparable between different cell states. Tag density depends on a number of factors including total number of tags sequenced, background modification levels of chromatin, and ChIP enrichment which are different for different samples. For that reason we only compare tag densities for various gene sets within the same sample.

\section{Presence/absence of a chromatin modification}

For each chromatin modification, a 500-bp region (either in the promoter or within the gene body) with maximal difference in the enrichment of modification between the constitutively expressed $(\mathrm{E} \rightarrow \mathrm{E})$ and constitutively silent $(\mathrm{S} \rightarrow \mathrm{S})$ genes was identified (Supplemental Table S1). A given chromatin modification is said to be present at a gene if the number of tags mapped within the iden- tified 500-bp window is statistically significant. To assess statistical significance, we modeled the distribution of tags throughout the genome as a Poisson process and calculated the number of reads necessary in 500-bp windows for a $P$-value threshold of $10^{-3}$. Since less than $80 \%$ of the genome length could be uniquely mapped using 25 bp reads, the background model assumed the genome size to be $2.46 \mathrm{~Gb}(80 \%$ of $3.08 \mathrm{~Gb})$. The number of tags necessary to satisfy the chosen $P$-value threshold is listed in Supplemental Table S1.

\section{Functional gene sets}

Genes related to certain biological processes were selected using MetaSearch software from the MetaCore package (Ekins et al. 2007) from GeneGo, Inc. (www.genego.com). The following GO biological process terms were used to select the genes: muscle: "muscle system process" or "muscle cell differentiation"; organ development: "liver development," "kidney development," "digestive tract morphogenesis," or "digestion"; "cell cycle"; and "metabolic process."

\section{MicroRNA promoter prediction}

For each modification (Pol II, H3K4me3, and H2A.Z), the tag density profile of tags at position $i$ in the genome is approximated using kernel density estimation (KDE) profiles. For Pol II, the tag density profile at position $i$ is given by

$$
S(i)=\frac{1}{h} \sum_{j=-i+3 h}^{i-3 h} K(j-i / h) \cdot C(j+75),
$$

where $h$ is the kernel density bandwidth (smoothing parameter, set to 20),

$$
K(x)=\frac{1}{\sqrt{2 \pi}} e^{\frac{-x^{2}}{2}}
$$

is the standard Gaussian kernel density function, and $C(x)$ is the sum of the number of $5^{\prime}$ read ends at position $x-80$ on the sense strand and position $x+80$ on the antisense strand. For H3K4me3 and H2A.Z, the tag density profiles at position $i$ are given by

$$
S(i)=\frac{1}{h} \sum_{j=-i+3 h}^{i-3 h} K(j-i / h) \cdot C(j+125)
$$

and

$$
S(i)=\frac{1}{h} \sum_{j=-i+3 h}^{i-3 h} K(j-i / h) \cdot C(j)
$$

respectively.

Putative TSS for each miRNA is predicted using a trace-back algorithm, which starts from the $5^{\prime}$ end of the pre-miRNA and traces back upstream looking for statistically significant peaks of Pol II, H3K4me3, and H2A.Z. Statistical significance of peaks was assessed by modeling the distribution of tags throughout the genome as a Poisson process and calculating the number of reads necessary in 400-bp windows for a $P$-value threshold of $10^{-6}$. For each modification, the KDE profiles are used to keep track of the tallest peak seen so far. The trace back stops if a peak has been found for all three modifications or $250 \mathrm{~kb}$ has been explored, whichever happens first. If a peak has been identified for all three modifications, the TSS for the miRNA is predicted as follows: If the Pol II peak colocalizes with peaks of H3K4me3 and/or H2A.Z, then the coordinate marking the Pol II peak is the TSS. Or else, if the H3K4me3 peak colocalizes with the H2A.Z peak, then the coordinate marking the H3K4me3 peak is the TSS. If neither of the 
above conditions holds, or if peaks for all three modifications have not been identified, then a prediction is not made. The region surrounding the predicted miRNA TSS is defined as the promoter. Predicted TSSs are expected to be within $500 \mathrm{bp}$ of the actual TSSs based on the TSS predictions of 1000 highly expressed genes in resting T cells (Supplemental Fig. S6). Predicted TSSs were manually inspected to filter out obvious false-positives by taking into account the three modifications used for prediction, as well as H3K36me3, H3K79me2, and the coordinates of known genes.

\section{$5^{\prime}-$ RACE}

Total RNA from resting and activated CD4+ T cell was prepared using RNeasy kit (Qiagen). Human placenta RNA was obtained from Clontech. Reverse transcription was performed using $1 \mu \mathrm{g}$ of RNA and a mixture of RT primers (Supplemental Fig. S7A; Supplemental Table S6) using a Smart RACE cDNA amplification kit (Clontech). PCR was performed using Universal primer (anneals to Clontech Smart Oligo) and individual RT primer. If no product was obtained, nested primer was used (Supplemental Table S6).

\section{Promoter reporter assay}

Approximately $650 \mathrm{bp}$ fragments surrounding putative promoters were amplified by PCR using high fidelity Phusion DNA polymerase (NEB) and cloned into KpnI/ BglII sites of pGL3 enhancer vector (Promega). Primers are listed in Supplemental Table S7. Jurkat cells were plated at $6 \times 10^{5}$ per well of six-well plate and transfected with $500 \mathrm{ng}$ of reporter construct, $50 \mathrm{ng}$ of pRLTK Renilla luciferase control plasmid, and $1 \mu \mathrm{g}$ of carrier DNA (pBSK) using Superfect reagent (Qiagen). Dual luciferase assay was performed $2 \mathrm{~d}$ later, and firefly luciferase signal was normalized by that of Renilla luciferase.

\section{Acknowledgments}

This work was supported by the Intramural Research Program of the National Heart, Lung and Blood Institute (NHLBI), National Institutes of Health. The gene expression analysis using the Affymetrix microarrays was performed by the Genomics Core Facility at NHLBI.

Author contributions: A.B., R.J., S.C., and K.Z. designed the study and wrote the paper; A.B., S.C., K.C. and T-Y.R. performed the experiments; R.J. analyzed the data with contributions from A.B. and D.E.S.

\section{References}

Barski A, Zhao K. 2009. Genomic location analysis by ChIP-seq. J Cell Biochem 107: 11-18.

Barski A, Cuddapah S, Cui K, Roh TY, Schones DE, Wang Z, Wei G, Chepelev I, Zhao K. 2007. High-resolution profiling of histone methylations in the human genome. Cell 129: 823-837.

Bartel DP, Chen CZ. 2004. Micromanagers of gene expression: The potentially widespread influence of metazoan microRNAs. Nat Rev Genet 5: 396-400.

Bernstein BE, Kamal M, Lindblad-Toh K, Bekiranov S, Bailey DK, Huebert DJ, McMahon S, Karlsson EK, Kulbokas EJ III, Gingeras TR, et al. 2005. Genomic maps and comparative analysis of histone modifications in human and mouse. Cell 120: 169-181.

Bernstein BE, Meissner A, Lander ES. 2007. The mammalian epigenome. Cell 128: 669-681.

Borchert GM, Lanier W, Davidson BL. 2006. RNA polymerase III transcribes human microRNAs. Nat Struct Mol Biol 13: 1097-1101.

Chambeyron S, Bickmore WA. 2004. Chromatin decondensation and nuclear reorganization of the HoxB locus upon induction of transcription. Genes \& Dev 18: 1119-1130.
Chandok MR, Farber DL. 2004. Signaling control of memory T cell generation and function. Semin Immunol 16: 285-293.

Chuang JC, Jones PA. 2007. Epigenetics and microRNAs. Pediatr Res 61: 24R29R.

Core LJ, Lis JT. 2008. Transcription regulation through promoter-proximal pausing of RNA polymerase II. Science 319: 1791-1792.

Crabtree GR. 1989. Contingent genetic regulatory events in T lymphocyte activation. Science 243: $355-361$.

Cuddapah S, Barski A, Cui K, Schones DE, Wang Z, Wei G, Zhao K. 2009. Native chromatin preparation and Illumina/Solexa Library Construction. Cold Spring Harb Protoc doi: 10.1101/pdb.prot5237.

Cui K, Zang C, Roh T-Y, Schones DE, Childs RW, Peng W, Zhao K. 2009. Chromatin signatures in multipotent human hematopoietic stem cells indicate the fate of bivalent genes during differentiation. Cell Stem Cell 4: 80-93.

Ekins S, Nikolsky Y, Bugrim A, Kirillov E, Nikolskaya T. 2007. Pathway mapping tools for analysis of high content data. Methods Mol Biol 356: 319-350.

Griffiths-Jones S, Grocock RJ, van Dongen S, Bateman A, Enright AJ. 2006. miRBase: MicroRNA sequences, targets and gene nomenclature. Nucleic Acids Res 34: D140-D144.

Guenther MG, Levine SS, Boyer LA, Jaenisch R, Young RA. 2007. A chromatin landmark and transcription initiation at most promoters in human cells. Cell 130: 77-88.

Guttman M, Amit I, Garber M, French C, Lin MF, Feldser D, Huarte M, Zuk O, Carey BW, Cassady JP, et al. 2009. Chromatin signature reveals over a thousand highly conserved large non-coding RNAs in mammals. Nature 458: 223-227.

Hager GL, Elbi C, Johnson TA, Voss T, Nagaich AK, Schiltz RL, Qiu Y, John S. 2006. Chromatin dynamics and the evolution of alternate promoter states. Chromosome Res 14: 107-116.

Heintzman ND, Stuart RK, Hon G, Fu Y, Ching CW, Hawkins RD, Barrera LO, Van Calcar S, Qu C, Ching KA, et al. 2007. Distinct and predictive chromatin signatures of transcriptional promoters and enhancers in the human genome. Nat Genet 39: 311-318.

Hsu F, Kent WJ, Clawson H, Kuhn RM, Diekhans M, Haussler D. 2006. The UCSC known genes. Bioinformatics 22: 1036-1046.

Huang da W, Sherman BT, Tan Q, Collins JR, Alvord WG, Roayaei J, Stephens R, Baseler MW, Lane HC, Lempicki RA. 2007. The DAVID Gene Functional Classification Tool: A novel biological module-centric algorithm to functionally analyze large gene lists. Genome Biol 8: R183. doi: $10.1186 / \mathrm{gb}-2007-8-9-\mathrm{r} 183$.

Janeway C. 2001. Immunobiology 5: The immune system in health and disease. Garland, New York.

Jenuwein T, Allis CD. 2001. Translating the histone code. Science 293: 1074 1080.

Kane LP, Lin J, Weiss A. 2000. Signal transduction by the TCR for antigen. Curr Opin Immunol 12: 242-249.

Kim TH, Barrera LO, Zheng M, Qu C, Singer MA, Richmond TA, Wu Y, Green RD, Ren B. 2005. A high-resolution map of active promoters in the human genome. Nature 436: 876-880.

Kolasinska-Zwierz P, Down T, Latorre I, Liu T, Liu XS, Ahringer J. 2009. Differential chromatin marking of introns and expressed exons by H3K36me3. Nat Genet 41: 376-381.

Kouzarides T. 2007. Chromatin modifications and their function. Cell 128: 693-705.

Krogan NJ, Dover J, Wood A, Schneider J, Heidt J, Boateng MA, Dean K, Ryan OW, Golshani A, Johnston M, et al. 2003. The Paf1 complex is required for histone $\mathrm{H} 3$ methylation by COMPASS and Dot1p: Linking transcriptional elongation to histone methylation. Mol Cell 11: 721729.

Lau NC, Lim LP, Weinstein EG, Bartel DP. 2001. An abundant class of tiny RNAs with probable regulatory roles in Caenorhabditis elegans. Science 294: $858-862$.

Lee Y, Kim M, Han J, Yeom KH, Lee S, Baek SH, Kim VN. 2004. MicroRNA genes are transcribed by RNA polymerase II. EMBO J 23: 4051-4060.

Li B, Carey M, Workman JL. 2007. The role of chromatin during transcription. Cell 128: 707-719.

Li J, Moazed D, Gygi SP. 2002. Association of the histone methyltransferase Set 2 with RNA polymerase II plays a role in transcription elongation. I Biol Chem 277: 49383-49388.

Marson A, Levine SS, Cole MF, Frampton GM, Brambrink T, Johnstone S, Guenther MG, Johnston WK, Wernig M, Newman J, et al. 2008. Connecting microRNA genes to the core transcriptional regulatory circuitry of embryonic stem cells. Cell 134: 521-533.

Mikkelsen TS, Ku M, Jaffe DB, Issac B, Lieberman E, Giannoukos G, Alvarez P, Brockman W, Kim TK, Koche RP, et al. 2007. Genome-wide maps of chromatin state in pluripotent and lineage-committed cells. Nature 448: 553-560.

Mohn F, Weber M, Rebhan M, Roloff TC, Richter J, Stadler MB, Bibel M, Schubeler D. 2008. Lineage-specific polycomb targets and de novo DNA

1750 Genome Research

www.genome.org 
methylation define restriction and potential of neuronal progenitors. Mol Cell 30: 755-766.

Muse GW, Gilchrist DA, Nechaev S, Shah R, Parker JS, Grissom SF, Zeitlinger J, Adelman K. 2007. RNA polymerase is poised for activation across the genome. Nat Genet 39: 1507-1511.

$\mathrm{Ng} \mathrm{HH}$, Robert F, Young RA, Struhl K. 2003. Targeted recruitment of Set 1 histone methylase by elongating Pol II provides a localized mark and memory of recent transcriptional activity. Mol Cell 11: 709719.

Pedersen I, David M. 2008. MicroRNAs in the immune response. Cytokine 43: $391-394$

Raisner RM, Hartley PD, Meneghini MD, Bao MZ, Liu CL, Schreiber SL, Rando OJ, Madhani HD. 2005. Histone variant H2A.Z marks the 5' ends of both active and inactive genes in euchromatin. Cell 123: 233-248.

Roh TY, Cuddapah S, Zhao K. 2005. Active chromatin domains are defined by acetylation islands revealed by genome-wide mapping. Genes \& Dev 19: $542-552$.

Roh TY, Cuddapah S, Cui K, Zhao K. 2006. The genomic landscape of histone modifications in human T cells. Proc Natl Acad Sci 103: 1578215787.

Ruthenburg AJ, Allis CD, Wysocka J. 2007. Methylation of lysine 4 on histone H3: Intricacy of writing and reading a single epigenetic mark. Mol Cell 25: 15-30.

Schones DE, Zhao K. 2008. Genome-wide approaches to studying chromatin modifications. Nat Rev Genet 9: 179-191.

Schones DE, Cui K, Cuddapah S, Roh TY, Barski A, Wang Z, Wei G, Zhao K. 2008. Dynamic regulation of nucleosome positioning in the human genome. Cell 132: 887-898.
Stallcup MR. 2001. Role of protein methylation in chromatin remodeling and transcriptional regulation. Oncogene 20: 3014-3020.

Steger DJ, Lefterova MI, Ying L, Stonestrom AJ, Schupp M, Zhuo D, Vakoc AL, Kim JE, Chen J, Lazar MA, et al. 2008. DOT1L/KMT4 recruitment and H3K79 methylation are ubiquitously coupled with gene transcription in mammalian cells. Mol Cell Biol 28: 2825-2839.

Vermaak D, Ahmad K, Henikoff S. 2003. Maintenance of chromatin states: An open-and-shut case. Curr Opin Cell Biol 15: 266-274.

Wang Z, Zang C, Rosenfeld JA, Schones DE, Barski A, Cuddapah S, Cui K, Roh TY, Peng W, Zhang MQ, et al. 2008. Combinatorial patterns of histone acetylations and methylations in the human genome. Nat Genet 40: $897-903$.

Wei G, Wei L, Zhu J, Zang C, Hu-Li J, Yao Z, Cui K, Kanno Y, Roh T-Y, Watford W, et al. 2009. Global mapping of histone H3 K4 and K27 trimethylation reveals specificity and plasticity in lineage fate determination of differentiating CD4+ T cells. Immunity 30: 155-167.

Weisbrod S. 1982. Active chromatin. Nature 297: 289-295.

Woods K, Thomson JM, Hammond SM. 2007. Direct regulation of an oncogenic micro-RNA cluster by E2F transcription factors. J Biol Chem 282: 2130-2134.

Zeitlinger J, Stark A, Kellis M, Hong JW, Nechaev S, Adelman K, Levine M, Young RA. 2007. RNA polymerase stalling at developmental control genes in the Drosophila melanogaster embryo. Nat Genet 39: 15121516.

Received January 7, 2009; accepted in revised form June 2, 2009. 
Genome Research 19: 1742-1751 (2009)

\section{Chromatin poises miRNA- and protein-coding genes for expression}

Artem Barski, Raja Jothi, Suresh Cuddapah, Kairong Cui, Tae-Young Roh, Dustin E. Schones, and Keji Zhao

Several errors in equations appear on page 1749 in the section MicroRNA Promoter Prediction. The corrected equations are provided below:

For Pol II, the tag density profile at position $i$ is given by

$$
S(i)=\frac{1}{h} \sum_{j=i-3 h}^{i+3 h} K[(i-j) / h] . C(j+75) .
$$

For H3K4me3 and H2A.Z, the tag density profiles at position $i$ are given by

and

$$
S(i)=\frac{1}{h} \sum_{j=i-3 h}^{i+3 h} K[(i-j) / h] . C(j+125)
$$

$$
S(i)=\frac{1}{h} \sum_{j=i-3 h}^{i+3 h} K[(i-j) / h] \cdot C(j)
$$

respectively.

The authors apologize for any confusion this may have caused.

Genome Research 15: 1746-1758 (2005)

\section{Comparing the human and chimpanzee genomes: Searching for needles in a haystack} Ajit Varki and Tasha K. Altheide

The URL in the last line of the footnote to Table 1 on page 1747 has been changed. The correct URL should now read:

http://carta.anthropogeny.org/moca/about. 


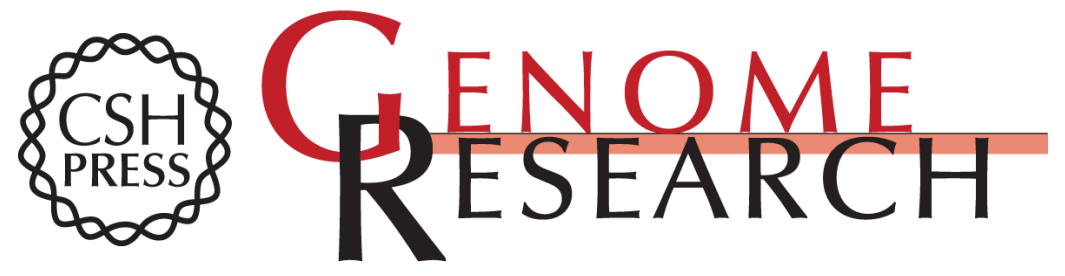

\title{
Chromatin poises miRNA- and protein-coding genes for expression
}

Artem Barski, Raja Jothi, Suresh Cuddapah, et al.

Genome Res. 2009 19: 1742-1751 originally published online August 27, 2009

Access the most recent version at doi:10.1101/gr.090951.109

\author{
Supplemental http://genome.cshlp.org/content/suppl/2009/08/28/gr.090951.109.DC2 \\ Material \\ Related Content Errata \\ Genome Res. December , 2009 19: 2343 \\ References This article cites 51 articles, 12 of which can be accessed free at: \\ http://genome.cshlp.org/content/19/10/1742.full.html\#ref-list-1 \\ Articles cited in: \\ http://genome.cshlp.org/content/19/10/1742.full.html\#related-urls

\section{License} \\ Email Alerting Receive free email alerts when new articles cite this article - sign up in the box at the \\ Service \\ top right corner of the article or click here.
}

\section{Affordable, Accurate} Sequencing. 